\title{
DESIGUALDADES DE GÉNERO EN LOS INVESTIGADORES PRINCIPALES DE LAS AYUDAS DEL PLAN NACIONAL SOBRE DROGAS, PUBLICACIONES DERIVADAS Y COMITÉS EDITORIALES DE REVISTAS SOBRE ADICCIONES
}

\section{GENDER INEQUALITIES IN MAIN RESEARCHERS OF GRANTS FROM PLAN NACIONAL SOBRE DROGAS, SUBSEQUENT PUBLICATIONS AND EDITORIAL COMMITTEES IN ADDICTION JOURNALS}

\begin{abstract}
Rafael Aleixandre-Benavent Unidad de Investigación e Información Social y Sanitaria-UISYS (CSIC-Universitat de València) Ingenio (CSIC-Universitat Politècnica de València)

Lourdes Castelló-Cogollos Unidad de Investigación e Información Social y Sanitaria-UISYS (CSIC-Universitat de València) Departamento de Sociología y Antropología Social. Universitat de València
\end{abstract}

Andrea Sixto-Costoya Unidad de Investigación e Información Social y Sanitaria-UISYS (CSIC-Universitat de València) Departamento de Historia de la Ciencia y Documentación. Universitat de València

Víctor Agulló-Calatayud Unidad de Investigación e Información Social y Sanitaria-UISYS (CSIC-Universitat de València) Departamento de Sociología y Antropología Social. Universitat de València

Adolfo Alonso-Arroyo

Unidad de Investigación e Información Social y Sanitaria-UISYS (CSIC-Universitat de València) Departamento de Historia de la Ciencia y Documentación. Universitat de València

Francisco Jesús Bueno-Cañigral Servicio de Drogodependencias (PMD/UPCCA-València). Concejalía de Salud y Consumo. Aj. València

Rut Lucas-Domínguez Unidad de Investigación e Información Social y Sanitaria-UISYS (CSIC-Universitat de València) Departamento de Historia de la Ciencia y Documentación. Universitat de València

David Melero-Fuentes Universidad Católica de Valencia "San Vicente Mártir"

Antonio Vidal-Infer

Unidad de Investigación e Información Social y Sanitaria-UISYS (CSIC-Universitat de València) Departamento de Historia de la Ciencia y Documentación. Universitat de València

Juan Carlos Valderrama-Zurián*

Unidad de Investigación e Información Social y Sanitaria-UISYS (CSIC-Universitat de València) Departamento de Historia de la Ciencia y Documentación. Universitat de València

*Contacto: UISYS (Universitat de València) Plaza Cisneros, 4 46003-València Juan.Valderrama@uv.es

Agradecimientos: Delegación de Gobierno para el Plan Nacional sobre Drogas (convocatoria 2016. Proyecto 2016/028). Ministerio de Sanidad Consumo y Bienestar Social. Servicio de Drogodependencias (PMD/UPCCA-València). Concejalía de Sanidad y Consumo. Ajuntament de València.

\section{Cómo citar este texto:}

Aleixandre-Benavent, R., Castelló-Cogollos, L., Sixto-Costoya, A. ,Agulló-Calatayud, V., Alonso-Arroyo, A., Bueno-Cañigral, F.J., Lucas-Domínguez, R., Melero-Fuentes, D., Vidal-Infer, A. y Valderrama-Zurián, J.C. (2022). Desigualdades de género en los investigadores principales de las Ayudas del Plan Nacional sobre Drogas, publicaciones derivadas y comités editoriales de revistas sobre adicciones. Health and Addictions / Salud y Drogas, 22(1), 


\section{Resumen}

Introducción: Se ha constatado una menor representación de las mujeres en la investigación sobre adicciones que puede mermar su aportación al área.

Objetivo: Analizar las diferencias de género en las distintas actividades relacionadas con la investigación en adicciones.

Material y método: Se calculó la representación de las mujeres españolas desde tres perspectivas: en las ayudas a proyectos de investigación financiados por el Plan Nacional Sobre Drogas (PNSD), en las publicaciones derivadas de estos proyectos, y en los comités editoriales (CE) de una selección de revistas sobre adicciones.

Resultados: El 26,9\% de investigadores principales de las ayudas del PNSD fueron mujeres. No se obsevaron diferencias estadísticamente significativas entre el sexo del investigador principal, el número de investigadores, su sexo y la colaboración con investigadores de otro sexo en las publicaciones. En tres revistas extranjeras sólo participa una mujer española como único miembro del CE (Alcohol and Alcoholism, Alcohol y Addictive Behaviors).

Conclusiones: Sólo una tercera parte de los investigadores principales de las ayudas del PNSD son mujeres y su representación no ha aumentado en los últimos años. La presencia de mujeres en los CE de las revistas españolas de adicciones es la quinta parte que la de los hombres y en las revistas extranjeras su representación es menor.

\section{Abstract}

Introduction: It has been observed that women are less represented in research on addictions, which may diminish their contribution to the area.

Purpose: To analyze the existence of gender inequality in different activities related to research on addictions.

Methods: The representation of Spanish women was calculated from three perspectives: in the grants to research projects funded by the Spanish Plan Nacional Sobre Drogas (PNSD), in the publications derived from these projects, and in the editorial committees (EC) of a selection of journals on addictions.

Results: Only $26.9 \%$ of the main researchers in the PNSD grants were women. No statistically significant differences were observed between the sex of the principal investigator, the number of researchers, their sex and the collaboration with researchers of another sex in the publications. In three foreign journals only one Spanish woman participates as the only member of the EC (Alcohol and Alcoholism, Alcohol and Addictive Behaviors).

Conclusions: Only a third of the main researchers of the PNSD grants are women and their representation has not increased in recent years. The presence of women in the EC of the Spanish journals of addictions is the fifth part that the one of the men and in the foreign journals their representation is smaller.

\section{Palabras clave}

Conductas adictivas; Desigualdades de género; Proyectos de investigación; Proyectos financiados; Revistas científicas; Sesgo de género

\section{Keywords}

Addictive behaviors; Gender inequalities; Research projects; Funded projects; Scientific journals; Gender bias 
Aleixandre-Benavent, R., Castelló-Cogollos, L., Sixto-Costoya, A. ,Agulló-Calatayud, V., Alonso-Arroyo, A., Bueno-Cañigral, F.J., Lucas-Domínguez, R., Melero-Fuentes, D., Vidal-Infer, A. y Valderrama-Zurián, J.C.

\section{a) Introducción}

Desde hace décadas, las sociedades contemporáneas intentan avanzar hacia una igualdad de oportunidades entre mujeres y hombres en todos los ámbitos de la vida. En la mayor parte de los países europeos, Japón, Nueva Zelanda, Australia y Norteamérica se ha logrado que mujeres y hombres tengan acceso a la educación primaria y secundaria sin discriminación y en igualdad de oportunidades (Lombardo, 2005; Bodelón, 2010). Por otra parte, la proporción de mujeres que terminan los estudios superiores en algunos países y áreas es incluso mayor que la de los hombres (Amrein et al., 2011; INEbase Educación, 2020; European Union, 2020). Sin embargo, a medida que se asciende en la escala de puestos de responsabilidad o liderazgo en los ámbitos profesionales y académicos, la representación de las mujeres es menor, fenómeno que se ha denominado «techo de cristal» (Lincoln et al., 2011; Brown y Diejman, 2010; Dobele et al., 2014; Matus-López y Gallego, 2015).

Se han publicado estudios que analizaron la presencia de la mujeres como autoras de los artículos científicos en varias áreas, entre ellas, algunas especialidades médicas (Aleixandre Benavent et al., 2007; Alonso Arroyo et al., 2008; Pastor Gosálbez et al., 2012; Diamond et al., 2016; Mayer et al., 2017; Yang et al., 2019), psicología (González Sala y Osca Lluch, 2018), sociología (González Alcaide et al., 2009; Agulló Calatayud y González Alcaide, 2011), información y documentación (Alonso Arroyo et al., 2010), educación (Álvarez González y Costa Aguirre, 2012) y periodismo (De-Miguel et al., 2017). Estos estudios han identificado una menor presencia de las mujeres como autoras de artículos científicos y esta disparidad se ha observado también en otras actividades académicas, como en los puestos de dirección en las asociaciones científicas y en las instituciones, así como en los comités editoriales (CE) de las revistas científicas y como editoras (Wenger, 2008; Amrein et al., 2011; Macaluso et al., 2016; Capdeville, 2019).

Las revistas científicas se consideran el principal medio de transmisión de conocimientos. Dirigir una revista científica o ser miembro de su comité editorial es un cargo y un reconocimiento que sólo puede alcanzarse tras una trayectoria investigadora y profesional de gran relieve (Alonso Arroyo et al., 2020). Estudios previos han informado que las mujeres están infrarrepresentadas en estos comités, así se han encontrado porcentajes muy bajos, como el $9 \%$ de mujeres en estudio sobre las revistas de Ortopedia (Rynecki et al., 2020), y el 11\% en Anestesiología (Capdeville, 2019). Entre los porcentajes más altos destacan el $41,7 \%$ encontrado en la revista JAMA Internal Medicine (Erren et al., 2014) o el 25\% en Especialidades Clínicas (Wenger, 2008). Recientemente, Alonso y colaboradores (2020) han encontrado porcentajes en las revistas pediátricas del 19\% para las editoras jefe y del 33\% de mujeres miembros del comité editorial, porcentajes que eran más elevados en revistas sobre enfermería pediátrica, fisioterapia, lactancia y nutrición.

En la actualidad, numerosas instituciones promueven iniciativas para lograr la equidad de género en los ámbitos académicos y profesionales. La Unión Europea ha publicado directrices para tener en cuenta la paridad de género en las actividades científicas, así como en el diseño y análisis de la investigación médica (European Commission, 2016; European Commission, 2020; Regensteiner et al., 2019). En los Estados Unidos de América, la American Association of University Women tiene como valores «promover la igualdad entre los géneros para las mujeres y las niñas mediante la investigación, la educación y la promoción» (American Association of University Women, 2020). Por otra parte, lograr la equidad de género es uno de los Objetivos de Desarrollo Sostenible del Milenio de la Organización Mundial de la Salud (OMS). El Objetivo 5, titulado «Promover la igualdad entre los géneros y la autonomía de la mujer, promueve «velar por la plena y efectiva participación de las mujeres y la igualdad de oportunidades de liderazgo a todos los niveles de la adopción de decisiones en la vida política, económica y pública» (United Nations, 2020).

Aunque los estudios de género han tenido un fuerte impulso en los últimos años, en el campo de las adicciones sólo hemos encontrado un artículo publicado en 2007 (González Alcaide et al., 2007), una tesis doctoral que analizaba el sexo de los miembros de los CE de las revistas de adicciones (Vidal-Infer, 2010), y dos trabajos recientes que investigaban este aspecto en las tesis doctorales (Castelló-Cogollos et al., 2019, Segarra-Saavedra y Villena-Alarcón, 2020).

El objetivo de este trabajo es caracterizar el papel de las mujeres en la investigación sobre adicciones desde tres perspectivas: a) la presencia de las mujeres como investigadoras principales (IP) en las ayudas a proyectos de investigación financiados por la Delegación del Gobierno para el Plan Nacional Sobre Drogas (PNSD); b) la participación 
de las mujeres en las publicaciones derivadas de los proyectos de investigación financiados; y c) la presencia de las mujeres españolas en los CE de una selección de revistas sobre adicciones.

\section{b) Método}

\section{b.1. Análisis de género de los investigadores principales (IP) de las ayudas del PNSD.}

EI PNSD viene convocando ayudas para el desarrollo de proyectos de investigación sobre adicciones desde el año 2000. Para el estudio de género de las personas beneficiarias de las ayudas se han analizado únicamente las concesiones de ayudas desde 2004 hasta 2016 (184 ayudas), ya que no se disponía de información sobre los IP en los años anteriores. La información sobre las características de las ayudas y sobre sus destinatarios es pública y está disponible en la web del PNSD (URL: https://pnsd.sanidad.gob.es/delegacionGobiernoPNSD/convocatoriaSubvenciones/ongs/proyeclnvestig.htm). De cada ayuda se recogió el año de concesión, nombre del IP y sexo del IP (mujer u hombre). Toda la información fue incluida en una base de datos de Microsoft Access.

\section{b.2. Presencia de investigadoras en las publicaciones derivadas de los proyectos de in- vestigación financiados por el PNSD}

Para caracterizar la participación de las mujeres en las publicaciones derivadas de los proyectos, se obtuvieron todos los artículos en los que constaba como fuente de financiación el PNSD y, en concreto, las ayudas para el desarrollo de proyectos de investigación sobre adicciones convocadas por el PNSD. El procedimiento de obtención de las publicaciones y un análisis de la producción se puede consultar en un trabajo previo (Aleixandre-Benavent, et al., 2020).

Se creó una base de datos con los registros correspondientes a las publicaciones distribuidos por campos. En el campo de cada registro en el que se consignaron los autores, se identificó el sexo de los autores de los trabajos. A continuación, se analizó la relación entre el sexo de los investigadores principales de los proyectos, número de colaboradores, sexo de los colaboradores, número de artículos derivados de los proyectos y presencia de mujeres en los artículos derivados. Para la comparación del sexo de los investigadores principales respecto a las variables cuantitativas, se utilizó la prueba t de student. Para la comparación del sexo de los investigadores principales con el sexo de los firmantes de los trabajos se utilizó la prueba de chi-cuadrado. El nivel de significación estadística se estableció para una $\mathrm{p}<0,05$

\section{b.3. Identificación de las revistas sobre adicciones en las que publican los autores espa- ñoles y representación de las mujeres españolas en los comités.}

Para identificar las revistas sobre conductas adictivas se realizó una búsqueda en las siguientes bases de datos bibliográficas: Science Citation Index Expanded (SCIE) y Social Science Citation Index (SSCI) incluídas en Web of Science Core Collection (WoS) de Clarivate Analytics; Scopus de Elsevier, que incluye Medline; Índices CSIC (bases de datos del Consejo Superior de Investigaciones Científicas). En la búsqueda se utilizaron tanto términos específicos de sustancias (por ejemplo, "éxtasis"), como generales (por ejemplo, "droga") y se seleccionarón los trabajos en los que figuraban instituciones españolas. La búsqueda se limitó al perido 2004-2016. Se estableció 2016 como límite, ya que este era el último año que estaban actualizadas las bases de datos cuando se realizaron las búsquedas. A continuación, los registros se distribuyeron por revistas de publicación y se identificaron las 15 revistas más productivas (con más de 50 trabajos) y se accedió a sus sitios en Internet para identificar las investigadoras españolas incluidas en sus CE. 
Aleixandre-Benavent, R., Castelló-Cogollos, L., Sixto-Costoya, A. ,Agulló-Calatayud, V., Alonso-Arroyo, A., Bueno-Cañigral, F.J., Lucas-Domínguez, R., Melero-Fuentes, D., Vidal-Infer, A. y Valderrama-Zurián, J.C.

\section{c) Resultados}

\section{c.1. Análisis de género de los investigadores principales de las Ayudas del PNSD.}

El $73,1 \%(n=79)$ de los IP beneficiarios de las ayudas eran hombres y el $26,9 \%(n=29)$ mujeres. El mayor porcentaje de mujeres fue en 2012, año en el que la distribución mujeres/hombres fue paritaria (50\%). Los menores porcentajes de mujeres fueron en $2007(7,1 \%, n=1)$ y en $2015(8,3 \%, n=1)$. No se aprecia una tendencia a la paridad en los cinco últimos años analizados, sino lo contrario, una disminución en el porcentaje de mujeres, que ha pasado del $50 \%$ ( $n=5$ ) en 2012 al 29,4\% ( $n=5)$ en 2016. La figura 1, que ofrece la imagen en espejo de los porcentajes de investigadores principales por sexo, permite apreciar la intensidad del denominado «efecto tijera» que se produce en las distribuciones por sexo en las que no existe paridad.

Figura 1. Distribución por sexo de los investigadores principales de las Ayudas de la Delegación del Gobierno para el Plan Nacional sobre Drogas para el desarrollo de proyectos de investigación sobre adicciones

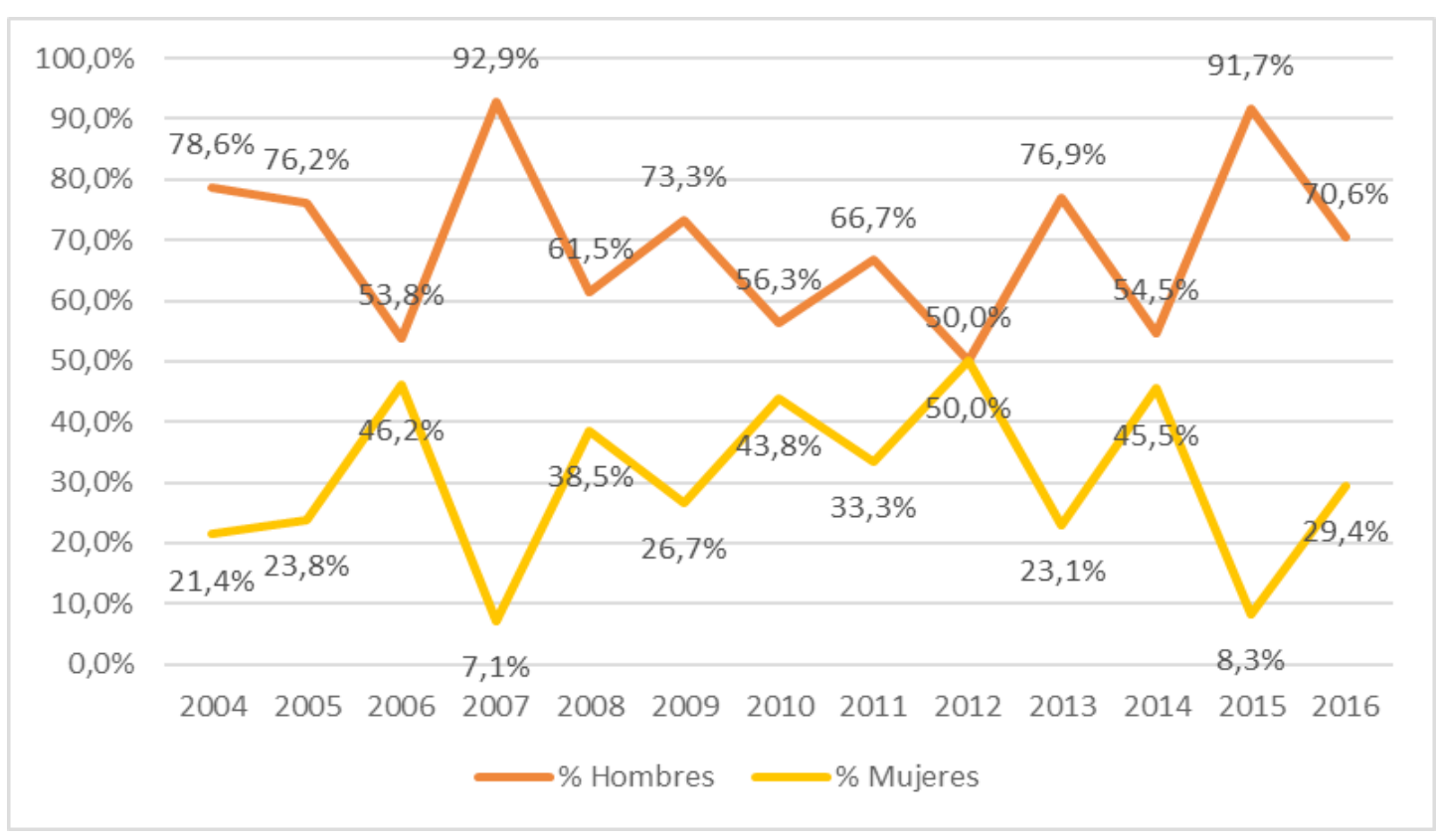

\section{c.2.Participación de mujeres en las publicaciones derivadas de los proyectos de inves- tigación}

Se identificó el sexo de los 2.504 autores que firmaron los 930 artículos derivados de los proyectos de investigación concedidos a 108 investigadores principales de los proyectos. El 47,9\% de los autores $(n=1.199)$ eran mujeres y el $52,1 \%(n=1.305)$ hombres. En el 95,3\% $(n=886)$ de los artículos firmaba al menos una investigadora. La media y desviación estándar del número investigadores con los que colaboraban los IP hombres era de 28,2 228,3 y la media y desviación estándar de los IP mujeres $25,3 \pm 24,15$. No se observaron diferencias estadísticamente significativas entre el sexo del IP, el número de autores y su sexo, y tampoco con el número de publicaciones derivadas o la presencia de al menos una mujer en los artículos. Sin embargo, se ha observado que las mujeres colaboran más con otras mujeres $(55,9 \%)$ que con hombres $(51,6 \%)\left(X^{2}=6,932, g \mid=1, p<0.009\right)$. 


\section{c.3. Análisis de género de los investigadores españoles en los comités editoriales de las revistas sobre adicciones}

Las 15 revistas dónde más han publicado los autores españoles se presentan en la tabla 1. De las revistas españolas, dos se han dejado de publicar (Trastornos Adictivos y Comunidad y Drogas) y otra, Drogalcohol, cambió su nombre en 1986 por Revista Española de Drogodependencias. El estudio se ha centrado, por tanto, en 12 revistas, de las que tres son españolas y 9 extranjeras.

El porcentaje de mujeres en los CE de las revistas españolas ha sido más alto en Revista Española de Drogodependencias $(31,9 \%, \mathrm{n}=26)$, seguida de Adicciones (29,1\%, $\mathrm{n}=25)$. En Health and Addiction / Salud y Drogas ha sido del $6,9 \%(n=2)$. No participa ninguna mujer en el CE de cuatro revistas extranjeras (Alcoholism: Clinical and Experimental Research; Addiction; European Addiction Research; Substance Use and Misuse) y en tres sólo participa una mujer española como único miembro de España en el CE (Alcohol and Alcoholism, Alcohol y Addictive Behaviors). La mujer que está presente en un mayor número de CE de revistas sobre adicciones es miembro en tres comités.

Tabla 1. Mujeres en los comités editoriales de las revistas más productivas en las que han publicado los investigadores españoles en adicciones

\begin{tabular}{|l|l|l|l|}
\hline Revista & No de miembros españoles & No de mujeres & \% de mujeres \\
& & & \\
\hline Adicciones & 86 & 25 & 29,1 \\
\hline Revista Española de Drogodependencias & 72 & 23 & 31,9 \\
\hline Health and Addictions / Salud y Drogas & 29 & 2 & 6,9 \\
\hline Drug and Alcohol Dependence & 2 & 0 & 0 \\
\hline $\begin{array}{l}\text { Alcoholism: Clinical and Experimental Re- } \\
\text { search }\end{array}$ & 0 & 0 & 0 \\
\hline Alcohol and Alcoholism & 1 & 1 & 100 \\
\hline Addiction & 0 & 0 & 0 \\
\hline Addiction Biology & 2 & 0 & 0 \\
\hline Alcohol & 1 & 1 & 100 \\
\hline Addictive Behaviors & 1 & 1 & 100 \\
\hline European Addiction Research & 0 & 0 & 0 \\
\hline Substance Use and Misuse & 0 & 0 & 0 \\
\hline
\end{tabular}

\section{d) Discusión y conclusiones}

Solo una tercera parte de los investigadores principales de las ayudas del PNSD son mujeres y no se ha observado que su representación haya aumentado en los últimos años. Esta baja representación no se justifica por una menor presencia como investigadoras, ya que el porcentaje de mujeres que cursan estudios superiores, doctorado o que acceden a la carrera académica es similar o incluso algo superior al de los hombres, tal y como puede observarse en el informe «Mujeres graduadas en educación superior» de 2014 del Instituto Nacional de Estadística (INEbase Educación, 2021).

La menor representación de las mujeres beneficiaras de ayudas a la investigación también se ha identificado en otros campos. Un estudio que analizó una muestra de premios de los Institutos Nacionales de Salud (NIH) en los Estados Unidos concluyó que el sesgo de género en la concesión de ayudas podían contribuir a la subrepresentación de las mujeres en la medicina académica (Biernat et al., 2020). En ese mismo país, otro trabajo que analizaba las 
Aleixandre-Benavent, R., Castelló-Cogollos, L., Sixto-Costoya, A. ,Agulló-Calatayud, V., Alonso-Arroyo, A., Bueno-Cañigral, F.J., Lucas-Domínguez, R., Melero-Fuentes, D., Vidal-Infer, A. y Valderrama-Zurián, J.C.

ayudas conseguidas en un departamento de urgencias concluía que sólo el $27 \%$ de los beneficiarios fueron mujeres y, aunque las becas de la propia institución se distribuyeron por igual entre hombres y mujeres, las mujeres recibieron menos becas del gobierno, la industria y de otras instituciones (Dubosh et al., 2019). Una investigación realizada en Canadá, concluyó que las mujeres que solicitaron subvenciones en varios institutos de investigación recibieron un porcentaje menor de subvenciones y un éxito significativamente menor en la adjudicación de personal de ayuda (Burns et al., 2019). En Europa, un estudio que analizaba el sexo de los proyectos financiados por el Biotechnology and Biological Sciences Research Council (BBSRC) del Reino Unido, concluyó que en 2014, las mujeres tuvieron una tasa de éxito del 17\% en comparación con el 44\% de los hombres (McAllister et al., 2016).

Para algunos investigadores, el sesgo de género en la dotación de subvenciones es una manifestación del sesgo de género histórico y sistémico en las instituciones académicas pues las mujeres, al obtener menos financiación, retrasan su progresión profesional y reducen sus oportunidades de publicar y, por lo tanto, su promoción profesional (Morgan, 2018). Ante estas evidencias, se ha sugerido que cuando se examinan las solicitudes para ser financiadas, se evalúe únicamente el proyecto científico y no al solicitante, eliminado así la referencia al sexo, ya que se ha demostrado que algunos revisores pueden introducir sesgos de género (Guglielmi, 2018).

En lo que respecta a la colaboración de hombres y mujeres en las publicaciones derivadas de los proyectos, no se han observado diferencias respecto al sexo del IP, el número de artículos derivados y la colaboración con investigadores de diferente sexo. Eso puede ser debido a que las mujeres IP son las más relevantes del área y, por ello, aglutinan a grupos de investigación y redes de colaboración con independencia del sexo de sus componentes.

La presencia de mujeres españolas en los CE de las revistas sobre adicciones apenas ha mejorado en una década. En 2010, Vidal-Infer ya indicaba que los autores procedentes de instituciones españolas participaban sólo en Drug and Alcohol Dependence, European Addiction Research y Addiction Biology, y que sólo la valenciana Consuelo Guerri formaba parte simultáneamente de dos CE (en Alcohol y Alcohol and Alcoholism) (Vidal-Infer, 2010). En nuestro trabajo, la presencia de mujeres en los CE de las revistas españolas de adicciones es solamente la quinta parte que la de los hombres. En las revistas extranjeras la situación es peor, ya que en cinco de las 11 revistas extranjeras no hay ningún miembro español en su CE, y en tres sólo participa una mujer como único miembro. En otros campos, como el biomédico, la situación es similar. Así, en un estudio que analizaba la proporción de mujeres en los CE de 63 revistas de 12 especialidades médicas diferentes, sólo el 16\% de los editores en jefe eran mujeres y en cinco especialidades no había una mujer que ocupara el puesto de editora jefe (Amrein et al., 2011). Esta baja representación se producía incluso en las especialidades en las que la proporción de mujeres era mayor que la de los hombres (Amrein et al., 2011). Posteriormente, otro estudio sobre seis revistas médicas de propósito general mostraba que la representación de las mujeres en los CE oscilaba entre el 22,2\% y el 41,7\% (Erren et al., 2014). Sin embargo, a pesar de una tendencia al alza en el número de mujeres, el aumento es lento y las proporciones de mujeres en los CE generalmente no reflejan su representación en sus respectivas especialidades (Capdeville et al., 2019).

La disminución de la representación de las mujeres a medida que se asciende en los cargos de responsabilidad se observó también en un estudio que analizaba su representación en las tesis doctorales sobre adicciones, directores y tribunales de tesis. La mitad de las tesis habían sido defendidas por mujeres, pero sólo el 32\% de las direcciones eran de mujeres, y en un porcentaje aún menor (26\%), las mujeres fueron miembros de los tribunales que las evaluaron (Castelló-Cogollos et al., 2019).

Los editores de las revistas pueden remediar la disparidad de género invitando a las mujeres a participar, ya sea en artículos editoriales o como revisoras, ya que el papel de revisor, realizado con profesionalidad, es un camino común para llegar a ser miembro de su CE (Wenger, 2008). En 2019, la revista The Lancet publicó un número temático sobre la mujer en la ciencia en el que hacía hincapié en la necesidad de producir cambios estructurales e institucionales para aumentar la representación de las mujeres y, además, adoptó la iniciativa \#LancetWomen con el objetivo de incorporar el género en las prácticas editoriales (The Lancet, 2019). Una de las medidas que puede favorecer llegar a esta paridad es el teletrabajo, ya que permite mantener reuniones a distancia independientemente de la localización geográfica y las mujeres que asumen compromisos familiares fuera del trabajo pueden adaptarse mejor (Robinson et al., 2010; Baecher-Lind, 2012). 
En España se han producido avances notables para lograr la igualdad, como lo demuestra el informe Mind the 100 Year Gap, publicado en 2020 por el Foro Económico Mundial, que analiza los progresos hacia la paridad entre los sexos en 153 países. En este informe, España ocupa el 8 lugar en el ranking de países con mayores progresos (World Economic Forum, 2020). Sin embargo, los resultados de nuestro análisis y los de otros estudios muestran que las desigualdades persisten, sobre todo en los puestos de responsabilidad y de liderazgo.

Los factores que influyen en que la representación de las mujeres sea menor que la de los hombres son diversos y multifactoriales y escapan del objetivo de este trabajo, pero se han señalado, entre otros, las interrupciones a las que está expuesta su carrera laboral y profesional producidas por la maternidad y, en ocasiones, por la mayor carga de responsabilidades familiares, así como diversas creencias que consideran que las mujeres son más proclives que los hombres a rechazar puestos de responsabilidad académica o profesional (Baecher-Lind, 2012; Cho et al., 2014). Según el Objetivo 5 de Desarrollo Sostenible de Naciones Unidas titulado «Lograr la igualdad entre los géneros y empoderar a todas las mujeres", las mujeres dedican en promedio unas tres veces más horas al día que los hombres a los cuidados no remunerados y al trabajo doméstico, lo que limita el tiempo disponible para el trabajo remunerado, la educación y el ocio, y refuerza aún más sus desventajas profesionales, académicas y socioeconómicas (United Nations, 2020).

Como limitaciones del estudio cabe señalar que las ayudas del PNSD no son la única vía de financiación de la investigación en adicciones en España. Por otra parte, sólo se han analizado una muestra de revistas y es posible que en otras la representación sea diferente. También es posible que se hayan cometido errores al asignar el sexo de los autores, ya que existen nombres que son iguales para hombres que para mujeres (como, por ejemplo, Andrea, que en español o euskera es nombre de mujer y en italiano de hombre). Por ello, futuras líneas de investigación podrían ampliar la muestra de ayudas a proyectos y de revistas analizadas, teniendo en cuenta las nuevas revistas digitales aparecidas en los últimos años, y seguir la evolución de la proporción de mujeres participantes. También sería de gran interés identificar los factores que obstaculizan que las mujeres alcancen la paridad en los tres aspectos estudiados en este trabajo.

Este trabajo permite concluir que solo una tercera parte de los investigadores principales de las ayudas del PNSD son mujeres y que esta baja representación no se justifica por una menor presencia como investigadoras. Por otra parte, la presencia de mujeres en los CE de las revistas españolas de adicciones es solamente la quinta parte que la de los hombres y en las revistas extranjeras apenas existen representantes españolas femeninas. En definitiva, la discriminación de las mujeres en las actividades académicas es un problema muy complejo que requiere la realización de estudios profundos que analicen todas las circunstancias, factores contextuales y aspectos sociológicos que pueden influir en la falta de paridad, incluyendo estudios que analicen la forma en que se organiza la ciencia como profesión y el sistema de recompensas de la productividad.

\section{e) Referencias}

American Association of University Women. AAUW's 2020 Gender Policy Agenda. Accessed 6/08/2020. Disponible en: https://www.aauw.org/resources/policy/aauws-2020-gender-policy-agenda

Agulló Calatayud, V y González Alcaide, G. (2011). La investigació sociològica valenciana a través de la revista Arxius de Ciències Socials. Arxius de sociologia, 24, 135-146.

Aleixandre Benavent, R., Agulló Calatayud, V., Alonso Arroyo A., Bueno-Cañigral, FJ., Castelló Cogollos, L., Lucas Domínguez, R., Melero Fuentes, D., Sixto Costoya, A., Vidal Infer, A., \& Valderrama Zurián, JC. (2021). Investigación española en las áreas de adicciones y de trastornos de la alimentación: análisis de la producción, colaboración e impacto científico (1962-2017). Revista Española de Documentación Científica, 44 (3). https://doi.org/10.3989/ redc.2021.3.1766

Aleixandre Benavent, R., Alonso Arroyo, A., González Alcaide, G., González de Dios, J., Sempere, AP., \& Valderrama 
Aleixandre-Benavent, R., Castelló-Cogollos, L., Sixto-Costoya, A. ,Agulló-Calatayud, V., Alonso-Arroyo, A., Bueno-Cañigral, F.J., Lucas-Domínguez, R., Melero-Fuentes, D., Vidal-Infer, A. y Valderrama-Zurián, J.C.

Zurián, JC. (2007). Análisis de género de los artículos publicados en Revista de Neurología durante el quinquenio 2002-2006. Revista de Neurología, 45(3), 137-144. https://doi.org/10.33588/rn.4503.2007213

Alonso Arroyo, A., González Alcaide, G., Bolaños Pizarro, M., Castelló Cogollos, L., Valderrama Zurián, JC., \& Aleixandre Benavent, R. (2008). Análisis de género de los trabajos publicados en la revista Actas Españolas de Psiquiatría (1999-2006). Actas Españolas de Psiquiatría, 36(6), 314-322.

Alonso Arroyo, A., Bolaños Pizarro, M., González Alcaide, G., Villamón, M., \& Aleixandre Benavent, R. (2010). Análisis de género, productividad científica y colaboración de las profesoras universitarias de ciencias de la salud en la Comunitat Valenciana (2003-2007). Revista Española de Documentación Científica, 33(4), 622-640. https://doi. org/10.3989/redc.2010.4.764

Alonso Arroyo, A., González de Dios, J., Aleixandre Agulló, J., \& Aleixandre Benavent, R. (2020). Gender inequalities on editorial boards of indexed pediatrics journals. Pediatric Research, Nov 25. https://doi.org/10.1038/s41390-020$\underline{01286-5}$

Álvarez González, B., \& Costa Aguirre, A.D. (2012). Goodbye sex equality. A new approach to the research on sex and gender. Educación XXI, 15, 61-86. https://doi.org/10.5944/educxx1.15.1.150

Amrein, K., Langmann, A., Fahrleitner-Pammer, A., Pieber, T.R., \& Zollner-Schwetz, I. (2011). Women underrepresented on editorial boards of 60 major medical journals. Gender Medicine, 8, 378-87. https://doi.org/10.1016/j. genm.2011.10.007

Baecher-Lind, L. (2012). Women in Leadership Positions Within. Obstetrics and Gynecology. Does the Past Explain the Present? Obstetrics and Gynecology, 120, 1415-1418. https://doi.org/10.1097/AOG.0b013e31829cf243

Biernat, M., Carnes, M., Filut, A., \& Kaatz, A. (2020). Gender, Race, and Grant Reviews: Translating and Responding to Research Feedback. Personality \& Social Psychology Bulletin, 46(1),140-154. https://doi. org/10.1177/0146167219845921

Bodelón, E. (2010). Las leyes de igualdad de género en España y Europa: ¿hacia una nueva ciudadanía? Anuario de Filosofía del Derecho, 26: 85-106.

Brown, E.R., \& Diejman, A.B. (2010). What Will I Be? Exploring Gender Differences in Near and Distant Possible Selves. Sex Roles, 63, 568-579. https://doi.org/10.1007/s11199-010-9827-x

Burns, K.E.A., Straus, S.E., Liu, K., Rizvi, L., \& Guyatt, G. (2019). Gender differences in grant and personnel award funding rates at the Canadian Institutes of Health Research based on research content area: A retrospective analysis. PLoS Medicine, 16(10), e1002935. https://doi.org/10.1371/journal.pmed.1002935

Capdeville, M. (2019). Don't Hold Your Breath-The Rise of Women on Journal Editorial Boards. Journal of Cardiothoracic and Vascular Anesthesia, 33, 3235-3238. https://doi.org/10.1053/i.jvca.2019.08.030

Castelló Cogollos, L., Bueno Cañigral, F.J., \& Valderrama Zurian, J.C. (2019). Bibliometric and academic network analysis of Spanish theses on drug dependence in the TESEO database. Adicciones, 31(4), 309-322. https://doi. org/10.20882/adicciones.1150

Cho, A.H., Johnson, S.A., Schuman, C.E., Adler, J.M., Gonzalez, O., Graves, S.J., Huebner, J.R., Marchant, D.B., Rifai, S.W., Skinner, I., \& Bruna, E.M. (2014). Women are underrepreseted on the editorial boards of journals in environmental biology and natural resource management. PeerJ, 2:e542. https://doi.org/10.7717/peerj.542 
De-Miguel, R., Hanitzsch, T., Parratt, S., \& Berganza, R. (2017). Mujeres periodistas en España: análisis de las características sociodemográficas y de la brecha de género. El Profesional de la Información, 26 (3), 497-506. https://doi. org/10.3145/epi.2017.may.16

Diamond, S.J., Thomas, C.R Jr., Desai, S., Holliday, E.B., Jagsi, R., Schmitt, C., \& Enestvedt, B.K. (2016). Gender Differences in Publication Productivity, Academic Rank, and Career Duration Among U.S. Academic Gastroenterology Faculty. Academic Medicine, 91(8),1158-63. https://doi.org/10.1097/ACM.0000000000000229

Dobele, A.R., Rundle-Thiele, S., \& Kopanidis, F. (2014). The cracked glass ceiling: equal work but unequal status. Higher Education Research \& Development, 33, 456-468. https://doi.org/10.1080/07294360.2013.841654

Dubosh, N.M., Boyle, K.L., Carreiro, S., Yankama, T., \& Landry A.M. (2019). Gender differences in funding among grant recipients in emergency medicine: A multicenter analysis. The American Journal of Emergency Medicine, 38(7), 1357-1361. https://doi.org/10.1016/i.ajem.2019.11.006

Erren, T.C., Groß, J.V., Shaw, D.M., \& Selle, B. (2014). Representation of women as authors, reviewers, editors in chief, and editorial board members at 6 general medical journals in 2010 and 2011. JAMA Internal Medicine, 174(4), 6335. https://doi.org/10.1001/jamainternmed.2013.14760

European Commission. (2016). Directorate-General for Research \& Innovation. H2020 programmes. Guidance on gender equality in Horizon 2020. Brussels: European Commission, 2016. Disponible en: https://eige.europa.eu/ sites/default/files/h2020-hi-guide-gender en.pdf

European Commission (2020). Horizon 2020. Promoting gender equality in research and innovation. Disponible en: https://ec.europa.eu/programmes/horizon2020/en/h2020-section/promoting-gender-equality-research-and-innovation

European Union (2020). Eurostat. Number of tertiary education students by level and sex, 2016. Disponible en: https://ec.europa.eu/eurostat/statisticsexplained/index.php?title=File:Number of tertiary education students by level and sex, 2016 (thousands) ET18.png

González Alcaide, G., Agulló Calatayud, V., Valderrama Zurián, J.C., \& Aleixandre Benavent, R. (2009). Participación de la mujer y redes de coautoría en las revistas españolas de Sociología. Revista Española de Investigaciones Sociológicas, 126, 153-166.

González Alcaide, G., Valderrama Zurián, J.C., Aleixandre Benavent, R., Alonso Arroyo, A., \& Miguel Dasit, A. (2007). Análisis de género de la producción científica española sobre drogodependencias en biomedicina (1999-2004). Adicciones, 19(1), 45-50. https://doi.org/10.20882/adicciones.322

González Sala, F., \& Osca Lluch, J. (2018). Desigualdad de género en órganos directivos y producción científica de las revistas iberoamericanas de psicología de mayor visibilidad internacional. Revista Española de Documentación Cientifica, 41 (3), e211. https://doi.org/10.3989/redc.2018.3.1506

Guglielmi, G. (2018). Gender bias goes away when grant reviewers focus on the science. Nature, 554(7690),14-15. https://doi.org/10.1038/d41586-018-01212-0

INEbase Educación. Porcentaje de hombres y mujeres graduados en educación superior en la UE (2014). Consultado el 19/4/2021. Disponible en https://www.ine.es/ss/Satellite?L=es_ES\&c=INESeccion_C\&cid=1259925481157\&p=1 254735110672\&pagename=ProductosYServicios/PYSLayout 
Aleixandre-Benavent, R., Castelló-Cogollos, L., Sixto-Costoya, A. ,Agulló-Calatayud, V., Alonso-Arroyo, A., Bueno-Cañigral, F.J., Lucas-Domínguez, R., Melero-Fuentes, D., Vidal-Infer, A. y Valderrama-Zurián, J.C.

Lincoln, Y.S., Lynham, S.A., \& Guba, E.G. (2011). Paradigmatic controversies, contra-dictions, and emerging confluences, revisited. En N.K. Denzin \& YS. The SAGE Hanbook of Qualitative Research. (97-128). SAGE Publications.

Lombardo, E. (2005). Integrating or setting the agenda? Gender mainstreaming in the European constitution-making processs, Social Politics, 12(3), 412-32. https://doi.org/10.1093/sp/jxi022

Macaluso, B., Larivière, V., Sugimoto, T., \& Sugimoto, C.R. (2016). Is Science Built on the Shoulders of Women? A Study of Gender Differences in Contributorship. Academic Medicine, 91(8), 1136-42. https://doi.org/10.1097/ ACM.0000000000001261

Matus López, M., \& Gallego, N. (2015). Techo de cristal en la Universidad. Si no lo veo no lo creo. Revista Complutense de Educación, 26, 611-626. https://doi.org/10.5209/rev RCED.2015.v26.n3.44491

Mayer, E.N., Lenherr, S.M., Hanson, H.A., Jessop, T.C., \& Lowrance W.T. (2017). Gender Differences in Publication Productivity Among Academic Urologists in the United States. Urology, 103:39-46. https://doi.org/10.1016/i.urology.2016.12.064

McAllister, D., Juillerat, J., \& Hunter, J. (2016). What stops women getting more grants? Nature, 529(7587), 466. https://doi.org/10.1038/529466d

Morgan, R., Hawkins, K., \& Lundine, J. (2018). The foundation and consequences of gender bias in grant peer review processes. Canadian Medical Association Journal, 190(16),E487-E488. https://doi.org/10.1503/cmaj.180188

Pastor Gosálbez, M.I., Belzunegui Eraso, A., \& Pontón Merino, P. (2012). Mujeres en sanidad: entre la igualdad y la desigualdad. Cuadernos de Relaciones Laborales, 30, 497-518. https://doi.org/10.5209/rev CRLA.2012.v30. $\underline{n} 2.40210$

Regensteiner J.G., Libby, A.M., Huxley, R., \& Clayton, J.A. (2019). Integrating sex and gender considerations in research: educating the scientific workforce. The Lancet Diabetes \& Endocrinology, 7, 248-250. https://doi. org/10.1016/S2213-8587(19)30038-5

Rynecki N.D., Krell E.S., Potter J.S., Ranpura A., \& Beebe K.S. (2020). How well represented are women orthopaedic surgeons and residents on major orthopaedic editorial boards and publications?. Clinical Orthopaedics and Related Research, 478(7), 1563-1568. https://doi.org/10.1097/CORR.0000000000000824

Robinson, S., Lecky, F., \& Mason, S. (2010). Editorial boards: where are all the women? European Journal of Emergency Medicine, 17(2), 61-2. https://doi.org/10.1097/MEJ.0b013e32833096cb

Segarra Saavedra, J., \& Villena Alarcón, E. (2020). Análisis bibliométrico, de redes sociales y citas en Google Académico de las tesis doctorales defendidas en España sobre adicciones (1976-2018). Health and Addictions/Salud y Drogas, 20, 2, 170-181. https://doi.org/10.21134/haaj.v20i2.551

The Lancet. (2019). Advancing women in science, medicine, and global health. Consultado el 27/3/2010. Disponible en: https://www.thelancet.com/lancet-women

United Nations. (2020). Sustainable Development Goal 5. Achieve gender equality and empower all women and girls. Consultado el 27/3/2010. Disponible en: https://sustainabledevelopment.un.org/sdg5 
Vidal Infer, A. (2019). Análisis de los artículos originales publicados en revistas específicas sobre drogodependencias incluidas en el Journal Citation Reports (2002-2006) [Tesis de doctorado, Universitat de València]. E-Archive: https://www.tesisenred.net/handle/10803/52191\#page=1

Wenger N.K. (2008). Women in leadership positions in the medical academic enterprise: what are the next steps? Archives of Internal Medicine, 168, 449-450. https://doi.org/10.1001/archinternmed.2007.127

World Economic Forum. Global Gender Gap Report 2020. Disponible en: http://www3.weforum.org/docs/WEF GGGR_2020.pdf

Yang, H.Y., Rhee, G., Xuan, L., Silver, J.K., Jalal, S., \& Khosa, F. (2019). Analysis of H-index in Assessing Gender Differences in Academic Rank and Leadership in Physical Medicine and Rehabilitation in the United States and Canada. American Journal of Physical Medicine \& Rehabilitation, 98(6),479-483. https://doi.org/10.1097/ PHM.0000000000001129 